\title{
Reconstructing 3D Volumes of Biological Specimens Using a Model Based Iterative Approach
}

Cheri M. Hampton ${ }^{1}$, Sherman J. Kinser ${ }^{2}$, Charles A. Bouman ${ }^{3}$ and Lawrence F. Drummy ${ }^{4}$

${ }^{1}$ UES, Inc., Dayton, Ohio, United States, ${ }^{2}$ High Performance Imaging, LLC, West Lafayette, Indiana, United States, ${ }^{3}$ Purdue University, West Lafayette, Indiana, United States, ${ }^{4}$ Air Force Research Laboratory, Wright-Patterson Air Force Base, Ohio, United States

Bright-field cryo electron tomography of biological specimens is a specialized type of tomography data that uses low dose/low signal-to-noise ratio images with limited angular range to reconstruct a threedimensional volume representation of the vitrified specimen. While filtered back-projection has remained one of the primary reconstruction methods, we demonstrate the usefulness of the model-based iterative method to generate 3D reconstructions that are significantly more robust, contain less noise, and allow for direct analysis and segmentation.

Previously published software [1] has been modified to accommodate low-dose, bright-field aligned tilt series as input and been made available through GitHub. Results and comparisons with traditional reconstruction methods will be demonstrated on a variety of biological specimens, such as vitrified cells and associated lipid structures. Figure 1 shows the difference between the filtered back-projection method and MBIR results. The cell imaged is E. coli expressing a construct for expression of the gvp1 cassette involved in the production of self-assembling, gas-filled vesicles. Due to their extreme contrast, these gasfillled vesicles are used as MRI contrasting agents [2].

MBIR (Model Based Iterative Reconstruction) is an approach to tomographic reconstruction that incorporates statistical models of the imaging system physics and the spatial characteristics of the imaging subject [3]. The projection image measurements are integrated with these models, and the reconstruction proceeds by optimizing the posterior distribution to find the most likely 3D volume given the measurements.

The relatively high computational load of MBIR has been greatly improved by structuring the optimization in a manner that maps efficiently onto current processors and results in a significant speedup [4,5]. This is critical for the TEM application in which increased detector resolution has allowed for highresolution reconstructions, but at the cost of having to solve a much larger optimization problem. It has also been observed in the current work that extending the reconstruction volume size beyond the scan ROI can significantly reduce artifacts in TEM reconstruction, for which it is susceptible from having sparse tilts and limited tilt range.

\section{References}

[1] Venkatakrishnan, S. V., Drummy, L. F., Jackson, M., De Graef, M., Simmons, J., \& Bouman, C. A. (2014). Model-based iterative reconstruction for bright-field electron tomography. IEEE Transactions on Computational Imaging, 1(1), 1-15. 

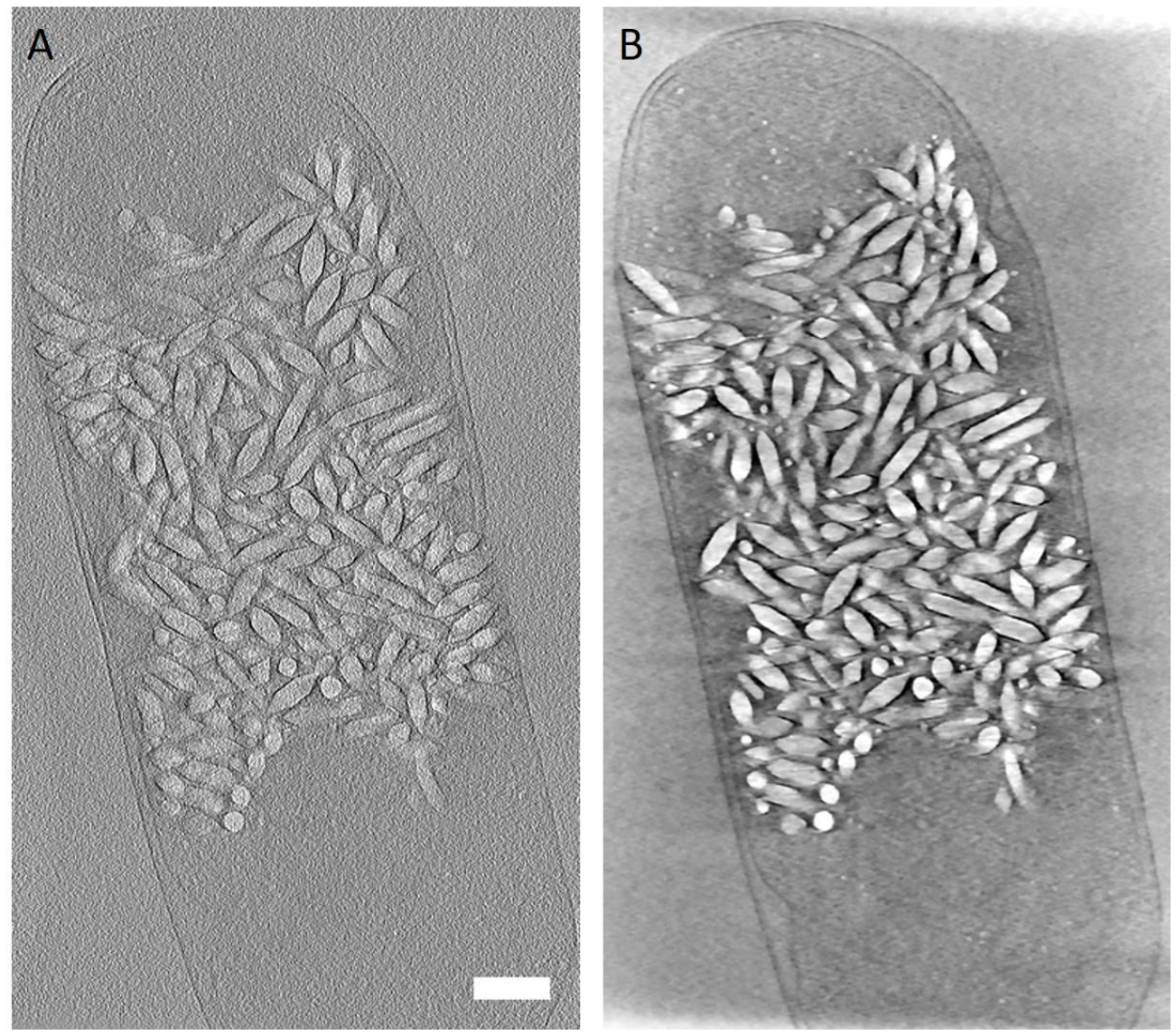

Figure 1. Filtered back-projection vs. MBIR generated volumes of a Gvp-1 expressing E. coli cell. Panel $\mathrm{A}$ is a central section of 3D volume produced by filtered back-projection using Etomo (3dmod, Boulder, CO) software. Panel B is same data reconstructed with the MBIR package. The MBIR prior model used here was a Q-generalized Gaussian Markov random field, or (QGGMRF), with a 3-D 10-point neighborhood, and parameters $(q, p, T)=(1.2,2.0,1.0)[6]$. Scale bar $=200 \mathrm{~nm}$.

[2] Farhadi, A., Ho, G. H., Sawyer, D. P., Bourdeau, R. W., \& Shapiro, M. G. (2019). Ultrasound imaging of gene expression in mammalian cells. Science, 365(6460), 1469-1475.

[3] C. A. Bouman and K. Sauer. A Unified Approach to Statistical Tomography Using Coordinate Descent Optimization. IEEE Transactions on Image Processing, 5(3), March 1996, 480--492.

[4] Xiao Wang, Amit Sabne, Putt Sakdhnagool, Sherman J Kisner, Charles A Bouman, and Samuel P Midkiff. Massively parallel 3D image reconstruction. In Proceedings of the International Conference for High Performance Computing, Networking, Storage and Analysis, SC '17. 2017.

[5] Super-Voxel Model Based Iterative Reconstruction (SVMBIR) [Computer software]. https://github.com/cabouman/svmbir

[6] Thibault, J. B., Sauer, K. D., Bouman, C. A., \& Hsieh, J. (2007). A three-dimensional statistical approach to improved image quality for multislice helical CT. Medical physics, 34(11), 4526-4544. 